\title{
Robotic Transaxillary Thyroid Lobectomy of a Follicular Neoplasm
}

\author{
Saleh Massasati, MD ${ }^{1}$, Salem Noureldine, MD, Rizwan Aslam, DO², and Emad Kandil, MD, FACS ${ }^{1}$ \\ ${ }^{1}$ Department of Surgery, Tulane University School of Medicine, New Orleans, LA; ${ }^{2}$ Department of Otolaryngology, \\ Tulane University School of Medicine, New Orleans, LA
}

\begin{abstract}
Purpose. Minimally invasive thyroid surgery using various techniques is well described. The purpose of this video is to show a robotic-assisted transaxillary right thyroid lobectomy for a follicular neoplasm with intraoperative nerve monitoring and stimulation of recurrent laryngeal nerve. Herein, we show our experience with the technique and its safety and feasibility.

Methods. We performed a right thyroid lobectomy on a 33-year-old patient using the da Vinci-Si-HD Surgical System. The operation was done via a single axillary incision, $5 \mathrm{~cm}$ in length. The flap creation time was approximately $26 \mathrm{~min}$. The robot docking time was $6 \mathrm{~min}$. The recurrent laryngeal nerve was identified and nerve
\end{abstract}

stimulation was used to stimulate with 0.5 milliamps. The operative console time was $21 \mathrm{~min}$. Total operative time was $69 \mathrm{~min}$.

Results. The procedure was successfully completed. Blood loss was minimal. Postoperative laryngoscopy showed intact and mobile bilateral vocal cords. There were no complications. Patient was discharged $4 \mathrm{~h}$ after surgery. Conclusions. Robotic transaxillary endoscopic gasless thyroid surgery with monitoring and stimulation of the RLN is feasible and safe. This technique eliminates a visible neck scar and affords excellent high definition optics of the cervical anatomy. This new technique can be accomplished on an outpatient basis.

Electronic supplementary material The online version of this article (doi:10.1245/s10434-011-2149-6) contains supplementary material, which is available to authorized users.

(C) Society of Surgical Oncology 2012

First Received: 10 September 2011;

Published Online: 10 March 2012

E. Kandil, MD, FACS

e-mail: ekandil@tulane.edu 